\title{
Variations in the Interplay of Intermetallic and Metal Chalcogenide Units in Organotin-Copper Selenide Clusters
}

Katharina Hanau, ${ }^{[a]}$ Niklas Rinn, ${ }^{[a]}$ and Stefanie Dehnen ${ }^{*[a]}$

[a] K. Hanau, Dr. N. Rinn, Prof. Dr. S. Dehnen

Fachbereich Chemie und Wissenschaftliches Zentrum für Materialwissenschaften (WZMW)

Philipps-Universität Marburg

Hans-Meerwein-Str. 4, 35043 Marburg (Germany)

E-mail: dehnen@chemie.uni-marburg.de

\section{Supporting Information}

1 Experimental Details

2 NMR Spectroscopy

3 X-ray Powder Diffraction

4 Micro X-ray Fluorescence Spectroscopy ( $\mu-X R F)$

5 Details of the X-ray Diffraction Measurements and Refinements

6 References for the Supporting Information 


\section{Experimental Details}

General: All syntheses were performed under exclusion of air and moisture using standard Schlenk techniques. All solvents were dried and freshly distilled prior to use. $\left[\left(\mathrm{R}^{1} \mathrm{Sn}\right)_{4} \mathrm{Se} 6\right],{ }^{1}\left(\mathrm{SiMe}_{3}\right)_{2} \mathrm{Se},{ }^{2}$ and $\left[\mathrm{CuCl}\left(\mathrm{PPh}_{3}\right)_{3}\right]^{3}$ were prepared according to literature procedures. Further chemicals were purchased from Sigma Aldrich.

Preparation of I: [(R $\left.\left.{ }^{1} \mathrm{Sn}\right)_{4} \mathrm{Se}_{6}\right](235 \mathrm{mg}, 0.175 \mathrm{mmol})$ and [CuCl$\left.\left(\mathrm{PPh}_{3}\right)_{3}\right](310 \mathrm{mg}, 0.350 \mathrm{mmol})$ were suspended in $40 \mathrm{~mL}$ of dichloromethane and $\left(\mathrm{SiMe}_{3}\right)_{2} \mathrm{Se}(157 \mathrm{mg}, 0.699 \mathrm{mmol})$ was added. After stirring at room temperature for $24 \mathrm{~h}$, a orange powder precipitated. Alternatively, only $20 \mathrm{~mL}$ of dichloromethane can be used, which speeds up the reaction as follows: After stirring for $30 \mathrm{~min}$, the reaction mixture changed from a yellow suspension to a clear, orange solution, and an orange powder started precipitating after $5 \mathrm{~h}$. The reaction mixture was stirred at room temperature for additional $14.5 \mathrm{~h}$ (18 $\mathrm{h}$ reaction time altogether). The bright orange powder (I) was filtered off and dried in vacuo. Layering the filtrate with $10 \mathrm{~mL}$ of $n$-hexane yielded single crystals of $\left[\left(\mathrm{CuPPh}_{3}\right)_{2}\left(\mathrm{SnCu}_{2}\right)\left\{\left(\mathrm{R}^{1} \mathrm{Sn}\right)_{2} \mathrm{Se}_{4}\right\}_{3}\right](\mathbf{B})$. I is metastable when stored in a dry argon atmosphere, yet turning (dark) red after $\sim 5 \mathrm{~d}$.

Preparation of 1: All of the powder that was isolated in the preparation step described above was suspended in $31 \mathrm{~mL}$ of dichloromethane and $\mathrm{N}_{2} \mathrm{H}_{4} \cdot \mathrm{H}_{2} \mathrm{O}\left(80 \%\right.$ in $\left.\mathrm{H}_{2} \mathrm{O}, 20 \mathrm{mg}, 0.320 \mathrm{mmol}\right)$ was added. After $10 \mathrm{~min}$, the powder had dissolved completely. The clear, orange solution was stirred for $16 \mathrm{~h}$, filtered, and layered with $10 \mathrm{~mL}$ of $n$-hexane. After $1 \mathrm{~d}$, yellow needles of 1 could be isolated ( $28 \mathrm{mg}, 19.5 \%$ compared to $\left.\left[\left(\mathrm{R}^{1} \mathrm{Sn}\right)_{4} \mathrm{Se}_{6}\right]\right)$. Due to the low solubility of $\mathbf{1}$, no NMR data could be obtained.

Alternative Preparation Method of 1: Alternatively, compound 1 can be prepared by extraction of the powder I with $10 \mathrm{~mL}$ of dichloromethane, filtration, addition of $\mathrm{N}_{2} \mathrm{H}_{4} \cdot \mathrm{H}_{2} \mathrm{O}\left(80 \%\right.$ in $\left.\mathrm{H}_{2} \mathrm{O}, 20 \mathrm{mg}, 0.320 \mathrm{mmol}\right)$, stirring for 16 hours, and layering with $10 \mathrm{~mL}$ of $n$-hexane.

Preparation of 2: Compound 2 was prepared following the alternative preparation method for the synthesis of compound 1, yet with the difference of layering with $10 \mathrm{~mL}$ of $n$-hexane immediately upon addition of $\mathrm{N}_{2} \mathrm{H}_{4} \cdot \mathrm{H}_{2} \mathrm{O}(80 \%$ in $\mathrm{H}_{2} \mathrm{O}, 20 \mathrm{mg}, 0.320 \mathrm{mmol}$ ). Single crystals of 2 were obtained in small yields as dark orange blocks, besides orange powder. Owing to the poor solubility of $\mathbf{2}$, sufficient amounts for NMR spectroscopy could not be obtained. Notably, the same batch affords compound $\mathbf{1}$ if following the other reaction procedure.

All our materials, including the products, were handled under Argon atmosphere. These compounds and our reported similar selenium clusters are especially sensitive to water. The single crystals themselves do also lose solvent molecules when handled in a solvent free atmosphere, and thereupon lose their crystallinity. 


\section{NMR Spectroscopy}

${ }^{1} \mathrm{H}$ NMR spectra of the reaction mixture producing powder I, or solutions of I, respectively, were recorded with a Bruker AV 500 spectrometer, and ${ }^{31}$ P NMR spectra were recorded with a Bruker AV III HD 300 spectrometer.

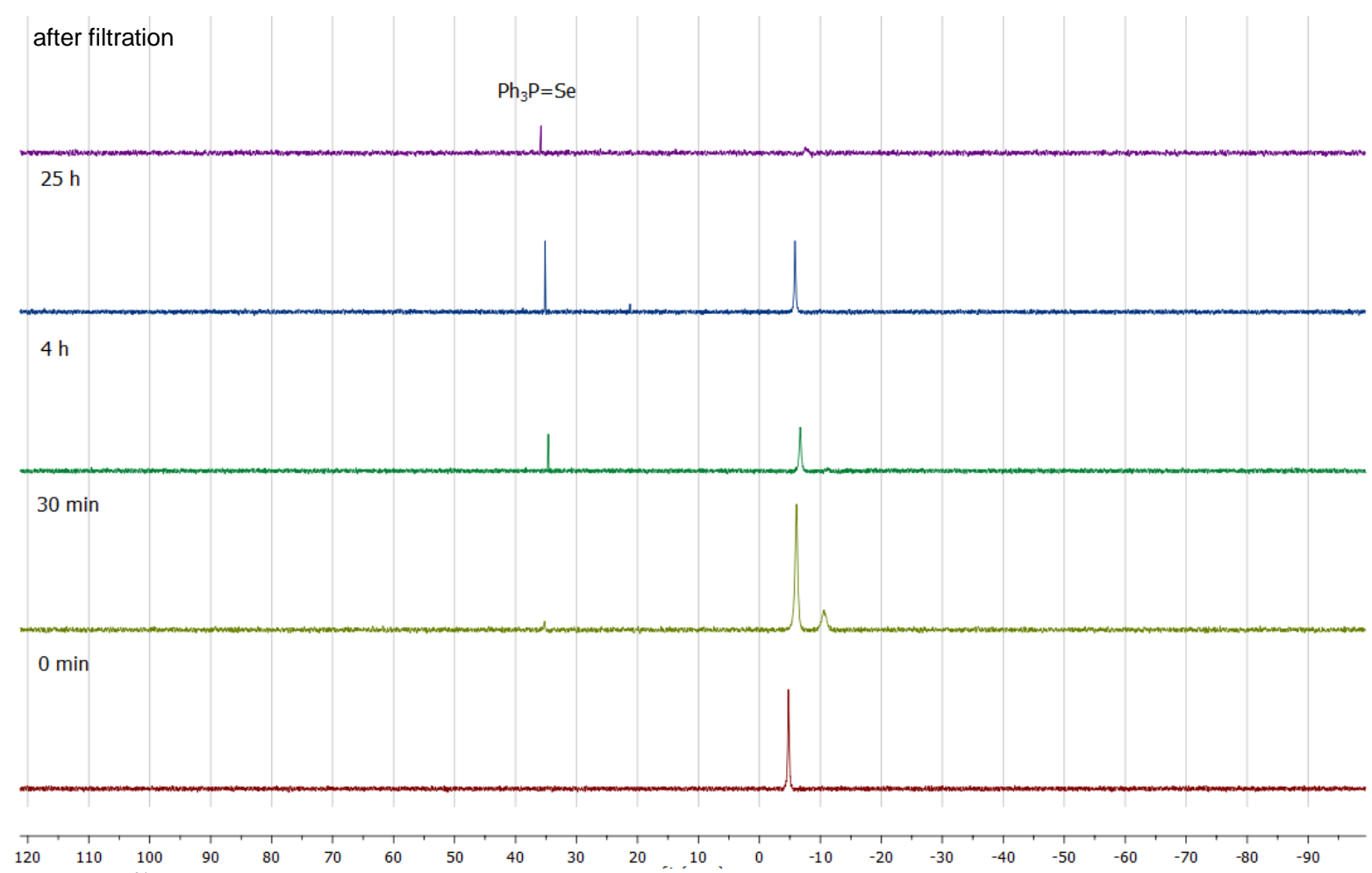

Figure S1. ${ }^{31} \mathrm{P}$ NMR spectra of the reaction yielding I at the beginning of the reaction, after $30 \mathrm{~min}, 4 \mathrm{~h}, 25 \mathrm{~h}$, and after work-up, respectively.

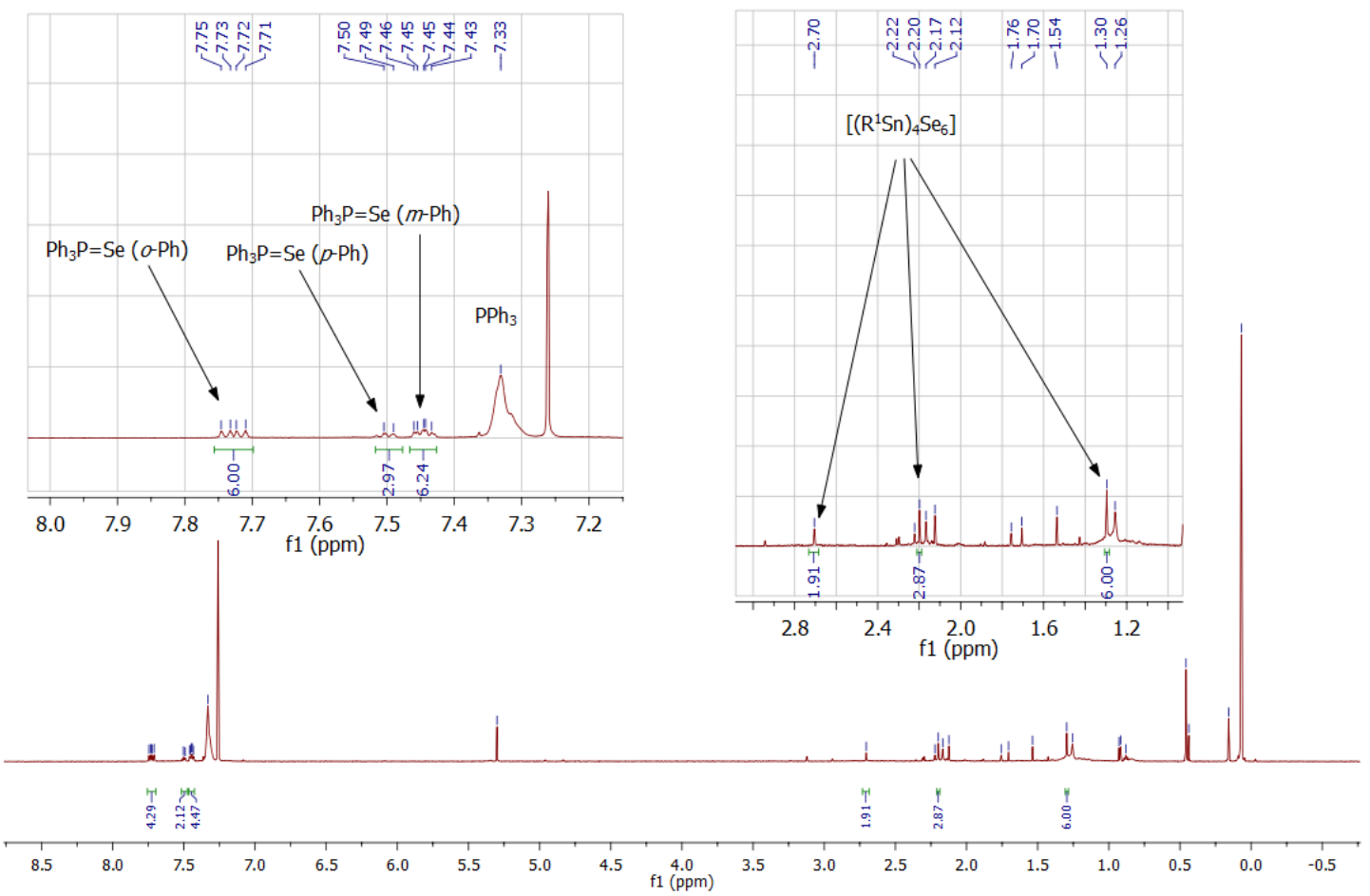

Figure S2. ${ }^{1} \mathrm{H}$ NMR spectrum of a solution of $\mathrm{I}$ in $\mathrm{CDCl}_{3}$. 


\section{X-ray Powder Diffraction}

In order to gain further insight into the composition of I, the powder was examined via X-ray powder diffraction and compared to the calculated powder diffractograms of $\mathbf{B}$ and 1. The diffractograms are shown in Figure S3. Powder Xray diffraction patterns were measured on a StadiMP diffractometer by Stoe equipped with a Mythen $1 \mathrm{~K}$ silicon strip detector and a Cu- $\mathrm{K}_{\alpha}(\lambda=1.54056 \AA)$ x-ray source. Samples were measured in transmission in a capillary.

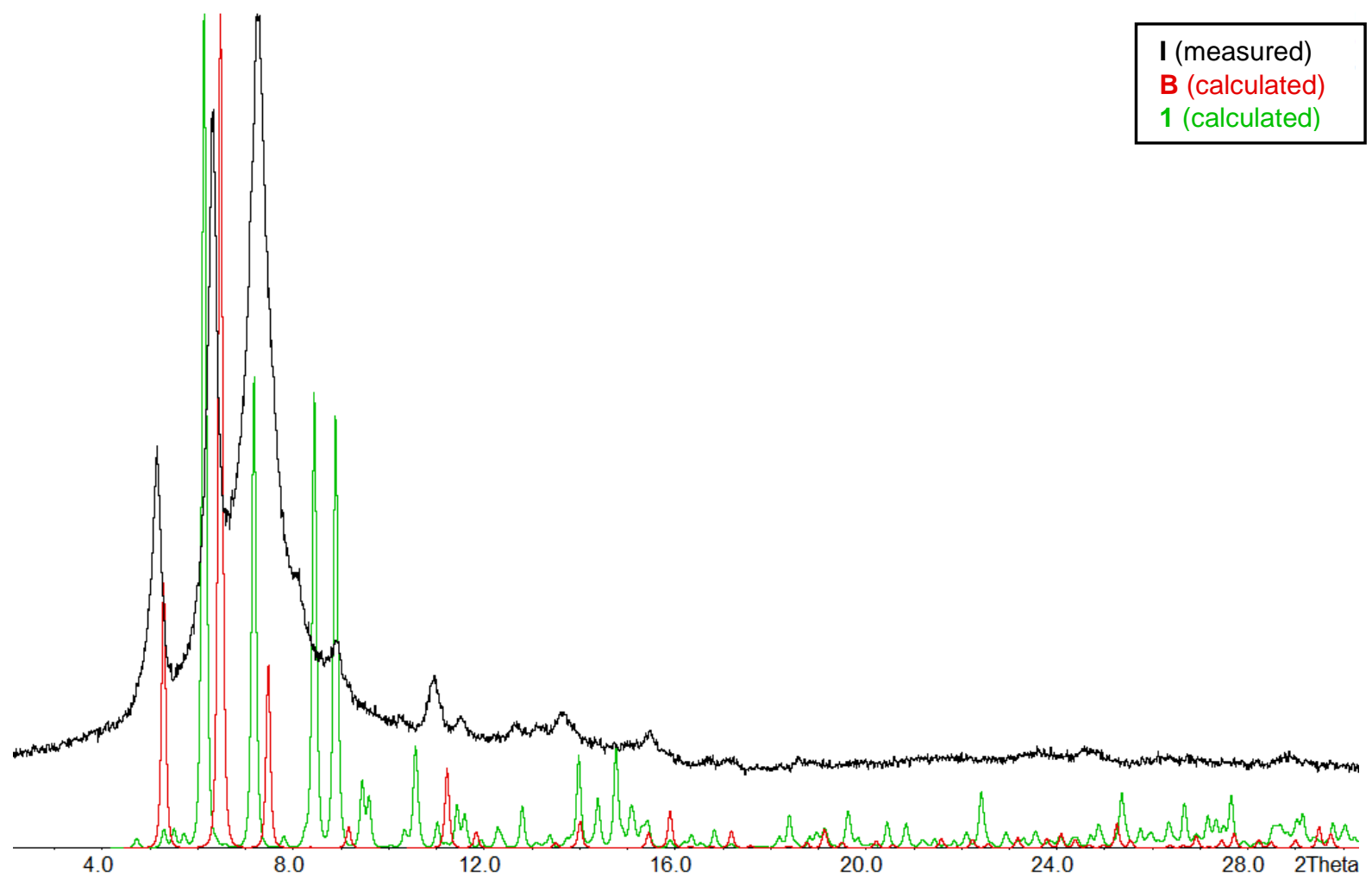

Figure S3. Comparison of the X-ray powder diffractograms of I, 1, and $\mathbf{B}$. The slight angular shift between the measured diffractogram of $\mathbf{I}$ and the calculated diffractogram of $\mathbf{B}$ is due to the single-crystal measurement of $\mathbf{B}$ being carried out at $100 \mathrm{~K}$ while the powder diffractogram was measured at $293 \mathrm{~K}$. 


\section{Micro X-ray Fluorescence Spectroscopy}

Micro X-ray fluorescence spectroscopy ( $\mu$-XRF) was carried out using a Bruker M4 Tornado spectrometer with an Rh target X-ray tube, poly-capillary optics and a Si drift detector.

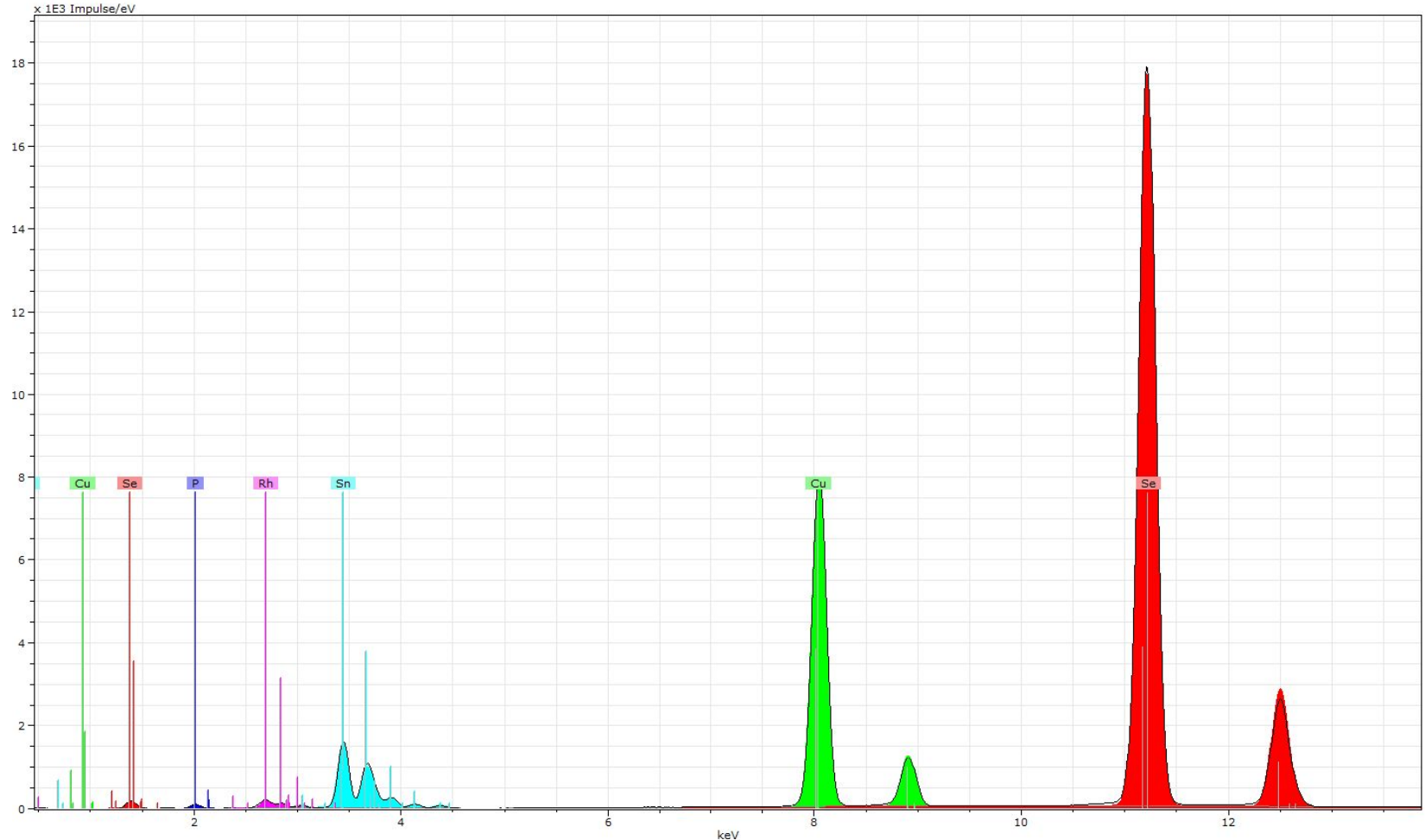

Figure S4. $\mu$-XRF spectrum of I. Values (\% found) for the elemental composition of P:Cu:Sn:Se was detected as follows: P: 5.57, Cu: 16.84, Sn: 26.57, Se: 51.03 , corresponding to atomic ratios of: 1:3:4.8:9.2. 


\section{Details of the X-ray Diffraction Measurements and Refinements}

\section{Crystal Structure of 1}

Crystals suitable for $X$-ray diffraction analyses were investigated with a STOE STADIVARI (1) diffractometer at $100 \mathrm{~K}$ using Cu- $K_{\alpha}$ radiation $(\lambda=1.54186)$ from an $X$-ray micro source with $X$-ray optics and a Pilatus $300 \mathrm{~K}$ Si hybrid pixel array detector. Upon spherical absorption correction and scaling (STOE LANA), the structure solution was performed by direct methods, followed by full-matrix-least-squares refinement against $F^{2}$, using SHELXT15, SHELXL15, and OLEX2 software. ${ }^{4-6}$

Hydrogen atom positions were calculated. Some of the co-crystallized solvent molecules could not be found/refined due to their disorder. They were thus detracted from the data by the back-Fourier-transform method. ${ }^{7}$ The voids are located at $(-0.071,0.293,-0.004),(0.071,0.7070 .004),(0.429,0.207,0.496)$, and $(0.571,0.793,0.504)$, have a volume of 182.2 each and contain $41.5,38.4,41.3$, and 38.4 electrons, respectively, which amounts to 2 molecules of either disordered dichloromethane or partially occupied $n$-hexane per formula unit.

The highest peak of residual electron density on the difference Fourier map $\left(4.437 \mathrm{e}^{-} / \AA^{3}\right)$ is found $1.030 \AA$ away from Sn5A.

A photograph of single crystals of 1 is shown in Figure S5 and excerpts of the crystal structure are shown in Figure S6.

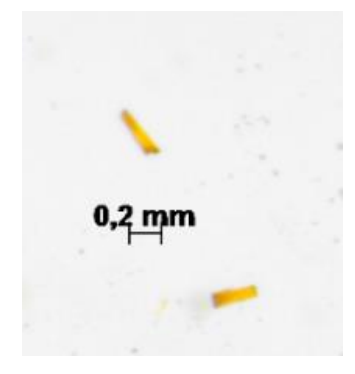

Figure S5. Photograph of single crystals of 1 picked for X-ray diffraction.
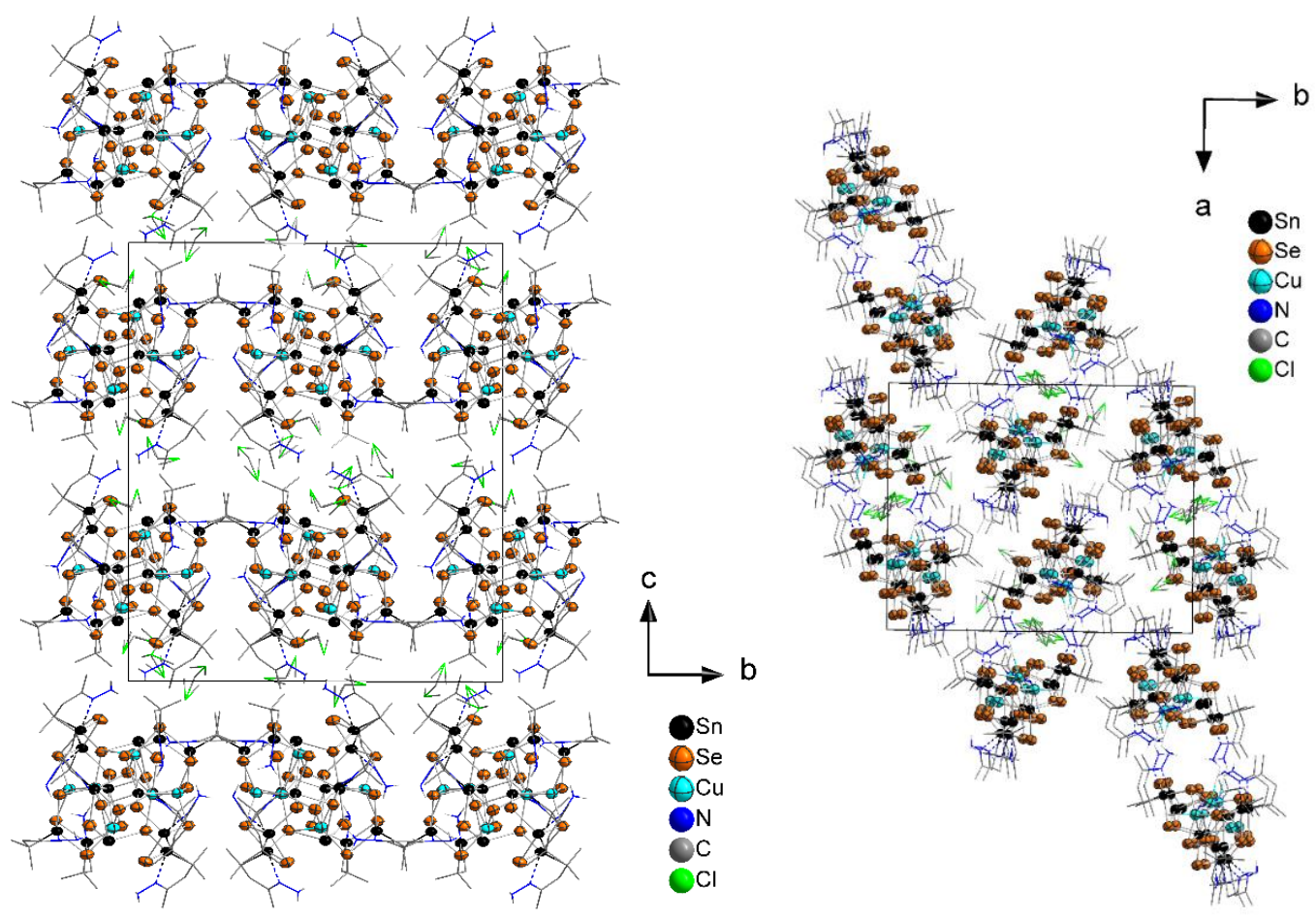

Figure S6. Excerpt of the crystal structure of 1 viewed along the a axis (left) and along the $c$ axis (right). 
Table S1. Crystallographic data and refinement results of 1.

\begin{tabular}{|c|c|}
\hline Compound & $\mathbf{1} \cdot 1.87 \mathrm{CH}_{2} \mathrm{Cl}_{2} \cdot 2 \mathrm{solv}$ \\
\hline Empirical Formula & $\mathrm{C}_{30} \mathrm{H}_{65} \mathrm{Cu}_{3} \mathrm{~N}_{10} \mathrm{Se}_{11} \mathrm{Sn}_{7}, 1.87 \mathrm{CH}_{2} \mathrm{Cl}_{2}$ \\
\hline Formula weight $/ \mathrm{g} \cdot \mathrm{mol}^{-1}$ & 2615.16 \\
\hline Crystal color and shape & yellow needle \\
\hline Crystal size $/ \mathrm{mm}^{3}$ & $0.032 \times 0.039 \times 0.191$ \\
\hline Crystal system & monoclinic \\
\hline Space group & $P 2_{1} / n$ \\
\hline$a / \AA$ & $19.9060(5)$ \\
\hline$b / \AA$ & $24.6240(4)$ \\
\hline$c / \AA$ & $28.8354(6)$ \\
\hline$\alpha /{ }^{\circ}$ & 90 \\
\hline$\beta /{ }^{\circ}$ & $92.446(2)$ \\
\hline$y /{ }^{\circ}$ & 90 \\
\hline$V / \AA^{3}$ & $14121.2(5)$ \\
\hline$z$ & 8 \\
\hline Measurement Temperature /K & 100 \\
\hline$\rho_{\text {calcd }} / \mathrm{g} \cdot \mathrm{cm}^{-3}$ & 2.460 \\
\hline$\mu_{(\mathrm{Cu} \mathrm{Ka})} / \mathrm{mm}^{-1}$ & 28.335 \\
\hline Absorption correction type & sphere \\
\hline Min./max. transmission & $0.074 / 0.464$ \\
\hline $2 \theta$ range / deg & $5.71-144.22$ \\
\hline No. of measured reflections & 216363 \\
\hline$R$ (int) & 0.1780 \\
\hline Independent Reflections & 22493 \\
\hline Independent Reflections $(1>2 \sigma(I))$ & 11006 \\
\hline No. of parameters & 1271 \\
\hline$R_{1}(/>2 \sigma(I)) / w R_{2}$ (all data) & $0.0868 / 0.2390$ \\
\hline$S$ (all data) & 0.889 \\
\hline Max. peak / hole / $\mathrm{e}^{-} \AA^{3}$ & $4.437 /-1.432$ \\
\hline
\end{tabular}


Table S2. Fractional Atomic Coordinates $\left(\times 10^{4}\right)$ and Equivalent Isotropic Displacement Parameters $\left(\AA^{2} \times 10^{3}\right)$ for EG062y. U(eq) is defined as $1 / 3$ of the trace of the orthogonalised $U_{I J}$ tensor.

\begin{tabular}{|c|c|c|c|c|c|c|c|c|c|}
\hline Atom & $x$ & $y$ & $z$ & $\mathrm{U}(\mathrm{eq})$ & Atom & $x$ & $y$ & $z$ & $U(e q)$ \\
\hline Sn1A & $6833.1(7)$ & $5276.6(6)$ & $7554.3(4)$ & $56.0(4)$ & C17A & $4776(12)$ & $5801(9)$ & $6118(8)$ & $74(7)$ \\
\hline Sn2A & $7843.5(8)$ & $4491.8(7)$ & $8644.5(5)$ & $67.4(4)$ & C18A & $4012(13)$ & $5724(11)$ & $6131(8)$ & $92(8)$ \\
\hline Sn3A & $8415.8(7)$ & $4106.0(6)$ & $7468.8(5)$ & $60.3(4)$ & C19A & $4915(12)$ & $6175(10)$ & $8853(7)$ & $74(7)$ \\
\hline Sn4A & $7878.2(8)$ & $6652.0(6)$ & $6594.1(4)$ & $59.5(4)$ & C20A & $5065(11)$ & $6670(10)$ & $9150(7)$ & $77(7)$ \\
\hline Sn5A & $6324.8(8)$ & $5846.1(6)$ & $6323.3(4)$ & $61.8(4)$ & $\mathrm{C} 21 \mathrm{~A}$ & $4789(12)$ & $5650(9)$ & $9189(6)$ & $71(7)$ \\
\hline Sn6A & $5809.9(7)$ & $5970.4(6)$ & $8467.2(4)$ & $57.6(4)$ & $\mathrm{C} 22 \mathrm{~A}$ & $4331(10)$ & $6247(9)$ & $8528(7)$ & $63(6)$ \\
\hline Sn7A & $7568.0(7)$ & $6036.0(6)$ & $8881.3(4)$ & $58.9(4)$ & C23A & $4497(10)$ & $6568(8)$ & $8084(6)$ & $52(5)$ \\
\hline Se1A & $7155.7(12)$ & $4278.9(9)$ & $7821.5(7)$ & $59.4(6)$ & C24A & $3945(11)$ & $6790(10)$ & $7777(7)$ & $75(7)$ \\
\hline $\mathrm{Se} 2 \mathrm{~A}$ & $8880.6(13)$ & $3978.1(11)$ & $8289.0(8)$ & $71.5(7)$ & $\mathrm{C} 25 \mathrm{~A}$ & $8072(11)$ & $6740(9)$ & $9182(8)$ & $68(6)$ \\
\hline Se3A & $8361.1(12)$ & $5476.7(9)$ & $8407.2(7)$ & $59.6(6)$ & C26A & $7620(13)$ & $7043(11)$ & $9502(8)$ & $90(8)$ \\
\hline Se4A & $8650.9(11)$ & $4998.9(9)$ & $7082.9(7)$ & $59.8(6)$ & C27A & $8270(12)$ & $7106(8)$ & $8802(7)$ & $66(6)$ \\
\hline Se5A & $6459.7(12)$ & $4919.5(10)$ & $6701.4(7)$ & $60.7(6)$ & C28A & $8715(12)$ & $6529(11)$ & $9464(7)$ & $81(7)$ \\
\hline Se6A & 7628.7(12) & $5852.1(10)$ & $6057.5(7)$ & $60.8(6)$ & C29A & $8587(13)$ & $6055(11)$ & $9744(7)$ & $74(7)$ \\
\hline Se7A & $6546.9(12)$ & $6677.3(10)$ & $6822.3(7)$ & $60.5(6)$ & C30A & $9056(11)$ & $5928(10)$ & $10159(7)$ & $78(7)$ \\
\hline Se8A & $8290.4(12)$ & $6589.1(10)$ & $7425.8(7)$ & $59.6(6)$ & Sn1B & $3130.8(7)$ & $4477.4(6)$ & $7452.2(4)$ & $57.5(4)$ \\
\hline Se9A & $5599.7(12)$ & $5235.4(9)$ & $7873.5(7)$ & $59.3(6)$ & Sn2B & 2115.3(8) & $5280.2(6)$ & $6376.2(5)$ & $67.1(4)$ \\
\hline Se10A & $6579.9(13)$ & $5473.8(11)$ & $9110.9(8)$ & $68.7(7)$ & Sn3B & $1546.0(8)$ & $5651.3(6)$ & $7553.8(5)$ & $61.7(4)$ \\
\hline Se11A & $6756.9(11)$ & $6621.5(9)$ & $8293.1(7)$ & $56.4(6)$ & Sn4B & $2090.0(8)$ & $3105.2(6)$ & $8421.1(4)$ & $58.7(4)$ \\
\hline Cu1A & $7125.6(15)$ & $6325.9(12)$ & $7549.8(9)$ & $58.4(8)$ & Sn5B & $3643.9(8)$ & $3902.5(6)$ & $8679.2(4)$ & $60.5(4)$ \\
\hline Cu2A & $8126.1(15)$ & $5643.5(12)$ & $7577.0(9)$ & $58.0(8)$ & Sn6B & $4141.4(7)$ & $3774.0(6)$ & $6529.1(4)$ & $57.2(4)$ \\
\hline Cu3A & $7649.0(16)$ & $5158.5(13)$ & $6674.0(10)$ & $66.6(8)$ & Sn7B & $2381.7(7)$ & $3733.3(6)$ & $6124.9(4)$ & $57.0(4)$ \\
\hline N1A & $9452(8)$ & $3772(7)$ & $7232(6)$ & $66(5)$ & Se1B & $2810.8(12)$ & $5480.1(10)$ & $7196.0(7)$ & $60.5(6)$ \\
\hline $\mathrm{N} 2 \mathrm{~A}$ & $10076(10)$ & $3936(10)$ & $7438(8)$ & $106(9)$ & $\mathrm{Se} 2 \mathrm{~B}$ & $1078.9(13)$ & $5784.6(11)$ & $6739.8(8)$ & $71.1(7)$ \\
\hline N3A & $9014(9)$ & $6588(7)$ & $6379(5)$ & $60(5)$ & Se3B & $1599.1(12)$ & 4297.3(9) & $6606.2(7)$ & $59.3(6)$ \\
\hline N4A & $9390(9)$ & $6113(6)$ & $6395(6)$ & $65(5)$ & Se4B & $1324.1(12)$ & $4755.6(10)$ & $7933.1(7)$ & $61.3(6)$ \\
\hline N5A & $5161(8)$ & $5868(7)$ & $6485(5)$ & $62(5)$ & Se5B & $1672.6(12)$ & $3170.4(9)$ & $7589.2(7)$ & $57.8(6)$ \\
\hline N6A & $4868(9)$ & $5927(7)$ & $6923(6)$ & $70(5)$ & Se7B & $2345.7(12)$ & $3904.5(10)$ & $8955.8(7)$ & $63.6(6)$ \\
\hline N7A & $5120(9)$ & $6608(8)$ & $8006(6)$ & $68(5)$ & Se6B & $3414.8(12)$ & 3073.8(9) & $8180.1(7)$ & $58.7(6)$ \\
\hline N8A & $5306(10)$ & $6904(9)$ & $7606(6)$ & $81(6)$ & Se8B & $3514.6(12)$ & $4832.4(10)$ & $8304.2(7)$ & $61.8(6)$ \\
\hline N9A & $8094(10)$ & $5748(8)$ & $9602(6)$ & $69(5)$ & Se9B & $4354.7(12)$ & $4508.1(10)$ & $7122.0(7)$ & $60.6(6)$ \\
\hline N10A & $7962(10)$ & $5268(9)$ & $9848(7)$ & $91(7)$ & Se10B & $3387.1(12)$ & $4254.2(11)$ & $5871.2(8)$ & $67.9(7)$ \\
\hline C1A & $8130(11)$ & $3437(9)$ & $7010(7)$ & $62(6)$ & Se11B & $3184.8(11)$ & $3136.6(9)$ & $6712.5(7)$ & $55.6(5)$ \\
\hline C2A & $7528(10)$ & $3593(9)$ & $6700(6)$ & $61(6)$ & Cu1B & $2835.1(16)$ & $3427.7(12)$ & $7461.3(9)$ & $59.9(8)$ \\
\hline C3A & $7950(11)$ & $2938(10)$ & $7342(7)$ & $78(7)$ & Cu2B & $1842.4(16)$ & $4113.9(13)$ & $7433.9(9)$ & $61.4(8)$ \\
\hline C4A & $8714(12)$ & $3271(10)$ & $6740(8)$ & $77(7)$ & Cu3B & $2330.7(17)$ & $4595.6(14)$ & $8342.7(11)$ & $70.7(9)$ \\
\hline C5A & $9431(12)$ & $3421(9)$ & $6924(7)$ & $66(6)$ & N1B & $517(9)$ & $5975(8)$ & $7797(7)$ & $73(5)$ \\
\hline C6A & $10006(13)$ & $3153(10)$ & 6733(8) & $84(7)$ & N2B & $-125(11)$ & $5709(12)$ & $7671(9)$ & $127(10)$ \\
\hline C7A & $8081(13)$ & $7409(9)$ & $6232(7)$ & $72(6)$ & N3B & $963(9)$ & $3164(8)$ & $8634(5)$ & $63(5)$ \\
\hline C8A & $7656(12)$ & $7871(9)$ & $6397(8)$ & $73(6)$ & N4B & $569(10)$ & $3635(9)$ & $8631(6)$ & $82(6)$ \\
\hline C9A & $7957(12)$ & $7326(9)$ & $5715(6)$ & $69(6)$ & N5B & $4812(9)$ & $3871(6)$ & $8513(5)$ & $56(4)$ \\
\hline C10A & $8811(13)$ & $7545(10)$ & $6356(9)$ & $82(7)$ & N6B & $5095(8)$ & $3816(8)$ & $8088(5)$ & $66(5)$ \\
\hline C11A & $9274(14)$ & $7060(13)$ & 6353(8) & $85(8)$ & N7B & $4834(9)$ & $3109(7)$ & $6987(5)$ & $62(5)$ \\
\hline C12A & $10052(13)$ & $7115(11)$ & $6351(10)$ & $100(9)$ & N8B & $4644(10)$ & $2811(7)$ & $7383(5)$ & $66(5)$ \\
\hline C13A & $5853(13)$ & $5872(11)$ & $5639(8)$ & $81(7)$ & N9B & $1841(10)$ & $4056(9)$ & $5407(6)$ & $76(6)$ \\
\hline C14A & $6143(15)$ & $5443(13)$ & $5343(8)$ & $107(10)$ & N10B & $1968(10)$ & $4547(7)$ & $5194(6)$ & $72(5)$ \\
\hline C15A & $5921(17)$ & $6411(13)$ & $5433(10)$ & 123(12) & C1B & $1823(11)$ & $6345(7)$ & $7980(7)$ & $60(6)$ \\
\hline C16A & $5076(15)$ & $5723(15)$ & $5674(8)$ & $119(12)$ & C2B & $2028(13)$ & $6787(8)$ & $7643(8)$ & $73(7)$ \\
\hline
\end{tabular}




\begin{tabular}{|c|c|c|c|c|c|c|c|c|c|}
\hline Atom & $x$ & $y$ & $z$ & $\mathrm{U}(\mathrm{eq})$ & Atom & $x$ & $y$ & $z$ & $\mathrm{U}(\mathrm{eq})$ \\
\hline C3B & $2431(12)$ & 6196(9) & $8316(8)$ & $80(7)$ & C25B & $1886(11)$ & $3024(8)$ & $5808(7)$ & $61(6)$ \\
\hline C4B & $1228(12)$ & $6523(9)$ & $8271(7)$ & $70(6)$ & $\mathrm{C} 26 \mathrm{~B}$ & $1647(12)$ & $2641(9)$ & $6173(7)$ & $73(7)$ \\
\hline C5B & $533(10)$ & $6354(8)$ & $8088(7)$ & $55(5)$ & C27B & 2398(14) & $2747(9)$ & $5506(8)$ & $94(9)$ \\
\hline C6B & $-58(11)$ & $6606(9)$ & $8299(7)$ & $68(6)$ & $\mathrm{C} 28 \mathrm{~B}$ & $1250(13)$ & 3253(9) & $5524(7)$ & $71(6)$ \\
\hline C7B & $1895(12)$ & $2361(8)$ & $8783(7)$ & $65(6)$ & C29B & $1366(12)$ & $3765(9)$ & $5259(7)$ & $64(6)$ \\
\hline $\mathrm{C} 8 \mathrm{~B}$ & $2005(11)$ & $2450(10)$ & $9300(7)$ & $70(6)$ & С $30 \mathrm{~B}$ & $896(11)$ & $3893(10)$ & $4854(7)$ & $77(7)$ \\
\hline C9B & $2374(12)$ & $1897(10)$ & $8633(8)$ & $79(7)$ & $\mathrm{Cl} 1$ & $9411(4)$ & $4767(3)$ & $9379(2)$ & $97(2)$ \\
\hline $\mathrm{C} 10 \mathrm{~B}$ & $1139(11)$ & $2200(10)$ & $8638(9)$ & $78(7)$ & $\mathrm{Cl} 2$ & $10466(5)$ & $5516(4)$ & $9153(3)$ & $136(3)$ \\
\hline C11B & $688(14)$ & $2695(11)$ & $8655(7)$ & $78(7)$ & C31 & $10003(14)$ & $4933(13)$ & $8955(8)$ & 109(9) \\
\hline $\mathrm{C} 12 \mathrm{~B}$ & $-84(13)$ & $2631(14)$ & $8597(12)$ & $130(13)$ & $\mathrm{Cl} 3$ & $9700(6)$ & $4205(4)$ & $5860(3)$ & 139(3) \\
\hline $\mathrm{C} 13 \mathrm{~B}$ & $4133(10)$ & 3819(9) & $9365(7)$ & $60(5)$ & $\mathrm{Cl} 4$ & $10481(4)$ & $5139(3)$ & $5628(2)$ & $97(2)$ \\
\hline C14B & $4166(14)$ & $3229(10)$ & $9504(9)$ & $92(8)$ & C32 & 9949(14) & $4845(8)$ & $6034(9)$ & $84(8)$ \\
\hline C15B & $3797(15)$ & $4166(14)$ & 9714(9) & $116(11)$ & $\mathrm{Cl} 5$ & $5707(9)$ & $7930(6)$ & $9656(7)$ & 181(9) \\
\hline $\mathrm{C} 16 \mathrm{~B}$ & $4886(11)$ & $4034(10)$ & $9329(7)$ & $69(6)$ & $\mathrm{Cl} 6$ & $6238(10)$ & $8462(8)$ & $10422(7)$ & 200(9) \\
\hline C17B & $5212(11)$ & $3960(9)$ & $8874(6)$ & $61(6)$ & C33 & $6390(20)$ & $8220(17)$ & $9908(10)$ & 109(9) \\
\hline C18B & $5937(11)$ & $4047(10)$ & $8842(7)$ & $70(6)$ & $\mathrm{Cl} 7$ & $6694(15)$ & $3645(12)$ & $10075(7)$ & $232(18)$ \\
\hline C19B & $5036(11)$ & $3597(8)$ & $6142(8)$ & $65(6)$ & $\mathrm{Cl} 8$ & $7178(12)$ & $4484(9)$ & 10572(6) & $149(10)$ \\
\hline $\mathrm{C} 20 \mathrm{~B}$ & $5190(12)$ & 4081(9) & $5833(8)$ & $75(7)$ & $\mathrm{C} 34$ & $7050(30)$ & $4210(18)$ & $10074(11)$ & $78(15)$ \\
\hline C21B & $4921(11)$ & $3087(9)$ & $5860(7)$ & $68(6)$ & $\mathrm{Cl} 9$ & $7050(13)$ & $4032(11)$ & $5091(8)$ & $225(15)$ \\
\hline C22B & $5615(10)$ & $3506(9)$ & $6506(6)$ & $61(6)$ & $\mathrm{Cl} 10$ & $8080(20)$ & $4386(8)$ & $5609(7)$ & $250(19)$ \\
\hline C23B & $5452(12)$ & $3173(9)$ & $6931(7)$ & $64(6)$ & C35 & $7280(20)$ & $4360(30)$ & $5546(19)$ & $180(40)$ \\
\hline C24B & $6002(10)$ & 2952(9) & $7230(7)$ & $64(6)$ & & & & & \\
\hline
\end{tabular}

Table S3. Selected bond lengths in $\mathbf{1}[\AA]$.

\begin{tabular}{|c|c|c|c|c|c|c|c|}
\hline Sn1A-Se1A & $2.646(3)$ & Sn5A-C13A & $2.15(2)$ & Sn1B-Se8B & $2.688(2)$ & Sn6B-Se10B & $2.647(3)$ \\
\hline Sn $1 A-S e 5 A$ & $2.687(2)$ & Sn6A-Se11A & $2.541(3)$ & Sn1B-Se9B & $2.654(3)$ & Sn6B-Se9B & $2.512(3)$ \\
\hline Sn1A-Se9A & $2.661(3)$ & Sn6A-Se9A & $2.514(3)$ & Sn1B-Cu1B & $2.651(3)$ & Sn6B-Se11B & $2.541(3)$ \\
\hline Sn1A-Cu1A & $2.649(3)$ & Sn6A-Se10A & $2.655(3)$ & Sn1B-Cu2B & $2.715(3)$ & Sn6B-N7B & $2.484(18)$ \\
\hline Sn1A-Cu2A & $2.726(3)$ & Sn6A-N7A & $2.442(18)$ & Sn1B-Cu3B & $3.093(3)$ & Sn6B-C19B & $2.19(2)$ \\
\hline Sn1A-Cu3A & $3.085(3)$ & Sn6A-C19A & $2.20(2)$ & Sn2B-Se1B & $2.733(3)$ & Sn7B-Se3B & $2.543(3)$ \\
\hline Sn2A-Se1A & $2.739(3)$ & Sn7A-Se11A & $2.707(3)$ & Sn2B-Se2B & $2.661(3)$ & Sn7B-Se10B & $2.512(3)$ \\
\hline Sn2A-Se2A & $2.663(3)$ & Sn7A-Se3A & $2.538(3)$ & Sn2B-Se3B & $2.722(3)$ & Sn7B-Se11B & $2.712(2)$ \\
\hline $\mathrm{Sn} 2 \mathrm{~A}-\mathrm{Se} 3 \mathrm{~A}$ & $2.733(3)$ & Sn7A-Se10A & $2.517(3)$ & Sn3B-Se1B & $2.793(3)$ & Sn7B-N9B & $2.427(18)$ \\
\hline Sn3A-Se1A & $2.779(3)$ & Sn7A-N9A & $2.395(18)$ & Sn3B-Se2B & $2.509(3)$ & Sn7B-C25B & $2.188(17)$ \\
\hline $\mathrm{Sn} 3 \mathrm{~A}-\mathrm{Se} 2 \mathrm{~A}$ & $2.522(3)$ & Sn7A-C25A & $2.16(2)$ & Sn3B-Se4B & $2.509(3)$ & Se3B-Cu2B & $2.457(3)$ \\
\hline $\mathrm{Sn} 3 \mathrm{~A}-\mathrm{Se} 4 \mathrm{~A}$ & $2.517(3)$ & Se11-Cu1A & $2.408(3)$ & Sn3B-N1B & $2.334(18)$ & Se4B-Cu2B & $2.400(4)$ \\
\hline Sn3A-N1A & $2.348(17)$ & Se3A-Cu2A & $2.454(3)$ & Sn3B-C1B & $2.163(16)$ & Se4B-Cu3B & $2.316(4)$ \\
\hline Sn3A-C1A & $2.17(2)$ & Se4A-Cu2A & $2.402(4)$ & Sn4B-Se5B & $2.510(2)$ & Se5B-Cu1B & $2.442(4)$ \\
\hline Sn4A-Se6A & $2.540(3)$ & Se4A-Cu3A & $2.307(4)$ & Sn4B-Se7B & $2.538(3)$ & Se5B-Cu2B & $2.393(4)$ \\
\hline Sn4A-Se7A & $2.758(3)$ & Se5A-Cu3A & $2.444(4)$ & Sn4B-Se6B & $2.757(3)$ & Se7B-Cu3B & $2.453(4)$ \\
\hline Sn4A-Se8A & $2.506(2)$ & Se6A-Cu3A & $2.464(4)$ & Sn4B-N3B & $2.356(19)$ & Se6B-Cu1B & $2.486(3)$ \\
\hline Sn4A-N3A & $2.375(19)$ & Se7A-Cu1A & $2.504(3)$ & Sn4B-C7B & $2.152(19)$ & Se8B-Cu3B & $2.435(4)$ \\
\hline Sn4A-C7A & $2.18(2)$ & Se8A-Cu1A & $2.448(4)$ & Sn5B-Se7B & $2.737(3)$ & Se11B-Cu1B & $2.406(3)$ \\
\hline Sn5A-Se5A & $2.538(3)$ & Se8A-Cu2A & $2.394(4)$ & Sn5B-Se6B & $2.527(3)$ & Cu1B-Cu2B & $2.599(4)$ \\
\hline Sn5A-Se6A & $2.737(3)$ & Cu1A-Cu2A & $2.604(4)$ & Sn5B-Se8B & $2.541(3)$ & Cu2B-Cu3B & $3.000(4)$ \\
\hline Sn5A-Se7A & $2.530(3)$ & Cu2A-Cu3A & $2.983(4)$ & Sn5B-N5B & $2.395(18)$ & & \\
\hline Sn5A-N5A & $2.383(17)$ & Sn1B-Se1B & $2.647(3)$ & Sn5B-C13B & $2.18(2)$ & & \\
\hline
\end{tabular}


Table S4. Selected bond angles in $1\left[^{\circ}\right]$.

\begin{tabular}{|c|c|c|c|c|c|}
\hline Se1A-Sn1A-Se5A & $90.97(8)$ & Se9A-Sn6A-Se11A & $115.02(8)$ & Se11A-Cu1A-Cu2A & $115.47(13)$ \\
\hline Se1A-Sn1A-Se9A & $94.58(8)$ & Se9A-Sn6A-Se10A & $102.64(9)$ & Se7A-Cu1A-Sn1A & $104.35(12)$ \\
\hline Se1A-Sn1A-Cu1A & $148.93(10)$ & N7A-Sn6A-Se11A & $83.7(4)$ & Se7A-Cu1A-Cu2A & $124.89(13)$ \\
\hline Se1A-Sn1A-Cu2A & $94.79(9)$ & N7A-Sn6A-Se9A & $91.1(4)$ & Se8A-Cu1A-Sn1A & $117.95(13)$ \\
\hline Se1A-Sn1A-Cu3A & $91.28(9)$ & N7A-Sn6A-Se10A & $166.2(4)$ & Se8A-Cu1A-Se7A & $101.00(12)$ \\
\hline Se5A-Sn1A-Cu2A & $110.76(9)$ & C19A-Sn6A-Se11A & $125.4(7)$ & Se8A-Cu1A-Cu2A & $56.46(11)$ \\
\hline Se5A-Sn1A-Cu3A & $49.51(8)$ & C19A-Sn6A-Se9A & $113.2(6)$ & Cu2A-Cu1A-Sn1A & $62.52(10)$ \\
\hline Se9A-Sn1A-Se5A & $94.64(8)$ & C19A-Sn6A-Se10A & $102.0(5)$ & Sn1A-Cu2A-Cu3A & $65.24(9)$ \\
\hline Se9A-Sn1A-Cu2A & $152.71(9)$ & C19A-Sn6A-N7A & $71.3(7)$ & Se3A-Cu2A-Sn1A & $96.23(11)$ \\
\hline Se9A-Sn1A-Cu3A & $143.81(9)$ & Se3A-Sn7A-Se11A & 108.63(8) & Se3A-Cu2A-Cu1A & $104.54(13)$ \\
\hline Cu1A-Sn1A-Se5A & $111.54(9)$ & Se10A-Sn7A-Se11A & $90.33(9)$ & Se3A-Cu2A-Cu3A & $145.57(15)$ \\
\hline Cu1A-Sn1A-Se9A & $104.20(10)$ & Se10A-Sn7A-Se3A & $110.75(10)$ & Se $4 A-C u 2 A-S n 1 A$ & $101.59(12)$ \\
\hline Cu1A-Sn1A-Cu2A & $57.95(10)$ & N9A-Sn7A-Se11A & $158.2(5)$ & $\mathrm{Se} 4 \mathrm{~A}-\mathrm{Cu} 2 \mathrm{~A}-\mathrm{Se} 3 \mathrm{~A}$ & $113.48(14)$ \\
\hline Cu1A-Sn1A-Cu3A & $87.96(9)$ & N9A-Sn7A-Se3A & $92.7(5)$ & Se4A-Cu2A-Cu1A & $139.46(14)$ \\
\hline Cu2A-Sn1A-Cu3A & $61.40(9)$ & N9A-Sn7A-Se10A & $85.8(5)$ & Se4A-Cu2A-Cu3A & $49.29(10)$ \\
\hline Se2A-Sn2A-Se1A & $86.71(8)$ & C25A-Sn7A-Se11A & $94.5(6)$ & Se8A-Cu2A-Sn1A & $117.01(13)$ \\
\hline Se2A-Sn2A-Se3A & $91.05(9)$ & C25A-Sn7A-Se3A & $111.2(6)$ & Se8A-Cu2A-Se3A & $108.58(13)$ \\
\hline Se3A-Sn2A-Se1A & $97.62(8)$ & C25A-Sn7A-Se10A & $133.5(6)$ & Se8A-Cu2A-Se4A & $117.94(14)$ \\
\hline Se2A-Sn3A-Se1A & $88.67(9)$ & C25A-Sn7A-N9A & $73.3(8)$ & Se8A-Cu2A-Cu1A & $58.48(11)$ \\
\hline Se4A-Sn3A-Se1A & $102.45(9)$ & Sn1A-Se1A-Sn2A & $100.42(9)$ & Se8A-Cu2A-Cu3A & $105.74(12)$ \\
\hline Se $4 A-S n 3 A-S e 2 A$ & $117.13(10)$ & Sn1A-Se1A-Sn3A & $104.32(9)$ & Cu1A-Cu2A-Sn1A & $59.54(10)$ \\
\hline N1A-Sn3A-Se1A & $167.8(5)$ & Sn2A-Se1A-Sn3A & $85.45(8)$ & Cu1A-Cu2A-Cu3A & $91.00(12)$ \\
\hline N1A-Sn3A-Se2A & $86.4(4)$ & $\mathrm{Sn} 3 \mathrm{~A}-\mathrm{Se} 2 \mathrm{~A}-\mathrm{Sn} 2 \mathrm{~A}$ & $92.42(9)$ & Se4A-Cu3A-Sn1A & $93.99(11)$ \\
\hline N1A-Sn3A-Se4A & $89.7(5)$ & Sn7A-Se3A-Sn2A & $95.55(9)$ & Se4A-Cu3A-Se5A & $139.46(16)$ \\
\hline C1A-Sn3A-Se1A & $97.0(6)$ & Cu2A-Se3A-Sn2A & $109.55(11)$ & Se4A-Cu3A-Se6A & $118.29(16)$ \\
\hline $\mathrm{C} 1 \mathrm{~A}-\mathrm{Sn} 3 \mathrm{~A}-\mathrm{Se} 2 \mathrm{~A}$ & $123.5(6)$ & Cu2A-Se3A-Sn7A & $109.73(11)$ & Se4A-Cu3A-Cu2A & $52.11(10)$ \\
\hline $\mathrm{C} 1 \mathrm{~A}-\mathrm{Sn} 3 \mathrm{~A}-\mathrm{Se} 4 \mathrm{~A}$ & $116.2(6)$ & Cu2A-Se4A-Sn3A & $102.78(11)$ & Se5A-Cu3A-Sn1A & $56.73(8)$ \\
\hline C1A-Sn3A-N1A & $76.6(7)$ & Cu3A-Se4A-Sn3A & $101.64(12)$ & Se5A-Cu3A-Se6A & $101.78(14)$ \\
\hline Se6A-Sn4A-Se7A & $89.88(9)$ & Cu3A-Se4A-Cu2A & $78.59(13)$ & Se5A-Cu3A-Cu2A & 109.94(13) \\
\hline Se8A-Sn4A-Se6A & $125.62(9)$ & Sn5A-Se5A-Sn1A & $96.84(9)$ & Se6A-Cu3A-Sn1A & $122.36(13)$ \\
\hline Se8A-Sn4A-Se7A & $92.96(8)$ & Cu3A-Se5A-Sn1A & $73.76(10)$ & Se6A-Cu3A-Cu2A & $110.31(14)$ \\
\hline N3A-Sn4A-Se6A & $87.3(4)$ & Cu3A-Se5A-Sn5A & $81.63(11)$ & Cu2A-Cu3A-Sn1A & $53.36(8)$ \\
\hline N3A-Sn4A-Se7A & $177.1(4)$ & Sn4A-Se6A-Sn5A & $89.81(8)$ & Se1B-Sn1B-Se8B & $90.42(8)$ \\
\hline N3A-Sn4A-Se8A & $88.3(4)$ & Cu3A-Se6A-Sn4A & $95.81(10)$ & Se1B-Sn1B-Se9B & $94.99(9)$ \\
\hline C7A-Sn4A-Se6A & $114.0(5)$ & Cu3A-Se6A-Sn5A & $77.34(10)$ & Se1B-Sn1B-Cu1B & $149.45(10)$ \\
\hline C7A-Sn4A-Se7A & $107.1(7)$ & Sn5A-Se7A-Sn4A & $89.56(8)$ & Se1B-Sn1B-Cu2B & $94.94(10)$ \\
\hline C7A-Sn4A-Se8A & $116.8(6)$ & Cu1A-Se7A-Sn4A & $77.28(10)$ & Se1B-Sn1B-Cu3B & $91.01(9)$ \\
\hline C7A-Sn4A-N3A & $74.6(8)$ & Cu1A-Se7A-Sn5A & $105.07(11)$ & Se8B-Sn1B-Cu2B & $110.88(9)$ \\
\hline Se5A-Sn5A-Se6A & $92.35(9)$ & Cu1A-Se8A-Sn4A & $83.26(10)$ & Se8B-Sn1B-Cu3B & $49.22(8)$ \\
\hline Se7A-Sn5A-Se5A & $118.05(8)$ & Cu2A-Se8A-Sn4A & $101.11(11)$ & Se9B-Sn1B-Se8B & $95.12(8)$ \\
\hline Se7A-Sn5A-Se6A & $90.58(9)$ & Cu2A-Se8A-Cu1A & $65.06(12)$ & Se9B-Sn1B-Cu2B & $152.03(10)$ \\
\hline N5A-Sn5A-Se5A & $91.3(4)$ & Sn6A-Se9A-Sn1A & $94.52(9)$ & Se9B-Sn1B-Cu3B & $143.93(9)$ \\
\hline N5A-Sn5A-Se6A & $174.8(4)$ & Sn7A-Se10A-Sn6A & $89.83(9)$ & Cu1B-Sn1B-Se8B & $111.26(9)$ \\
\hline N5A-Sn5A-Se7A & $91.1(4)$ & Sn6A-Se11A-Sn7A & $88.16(8)$ & Cu1B-Sn1B-Se9B & $103.82(10)$ \\
\hline C13A-Sn5A-Se5A & $117.1(7)$ & Cu1A-Se11A-Sn6A & $104.01(11)$ & Cu1B-Sn1B-Cu2B & $57.92(10)$ \\
\hline C13A-Sn5A-Se6A & $97.2(7)$ & Cu1A-Se11A-Sn7A & $101.56(11)$ & Cu1B-Sn1B-Cu3B & $87.77(10)$ \\
\hline C13A-Sn5A-Se7A & $123.8(7)$ & Se11A-Cu1A-Sn1A & $102.42(12)$ & Cu2B-Sn1B-Cu3B & $61.81(9)$ \\
\hline C13A-Sn5A-N5A & $77.8(8)$ & Se11A-Cu1A-Se7A & $119.64(14)$ & Se2B-Sn2B-Se1B & $87.00(8)$ \\
\hline Se11-Sn6A-Se10A & $91.02(9)$ & Se11A-Cu1A-Se8A & $112.12(13)$ & Se2B-Sn2B-Se3B & $90.68(9)$ \\
\hline
\end{tabular}




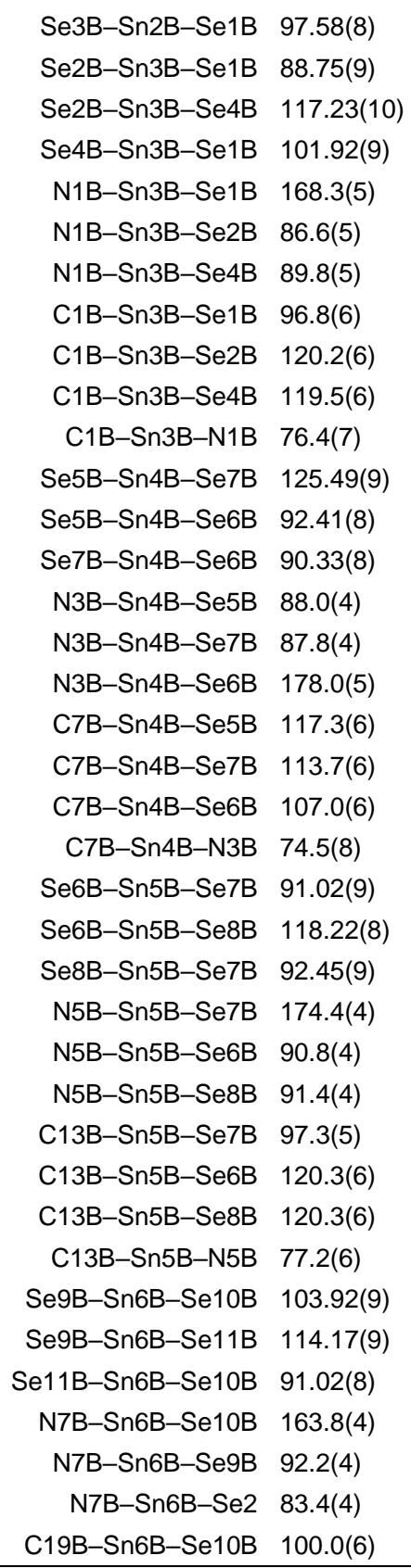

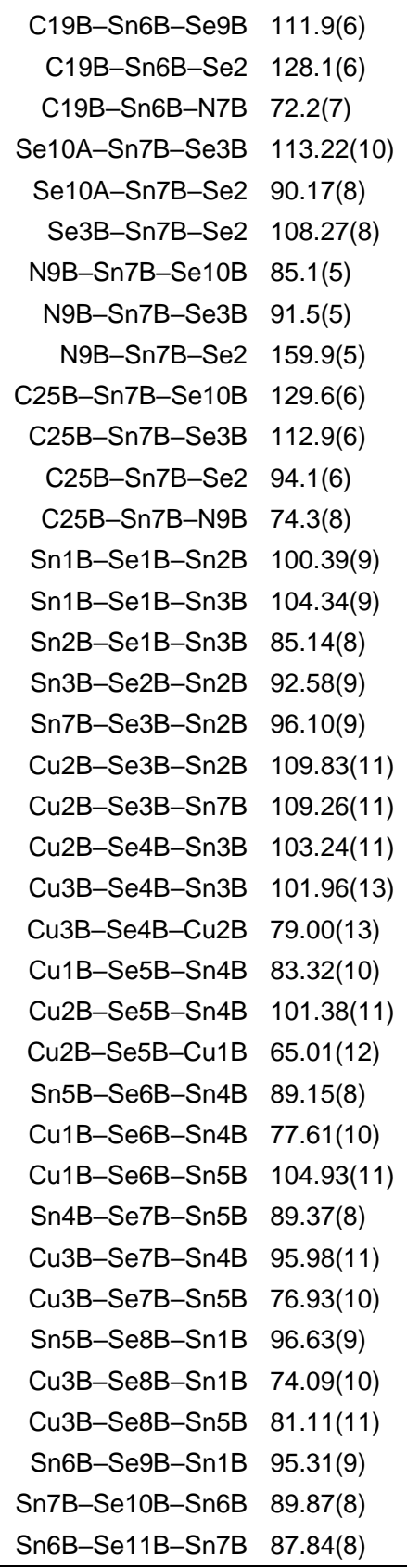

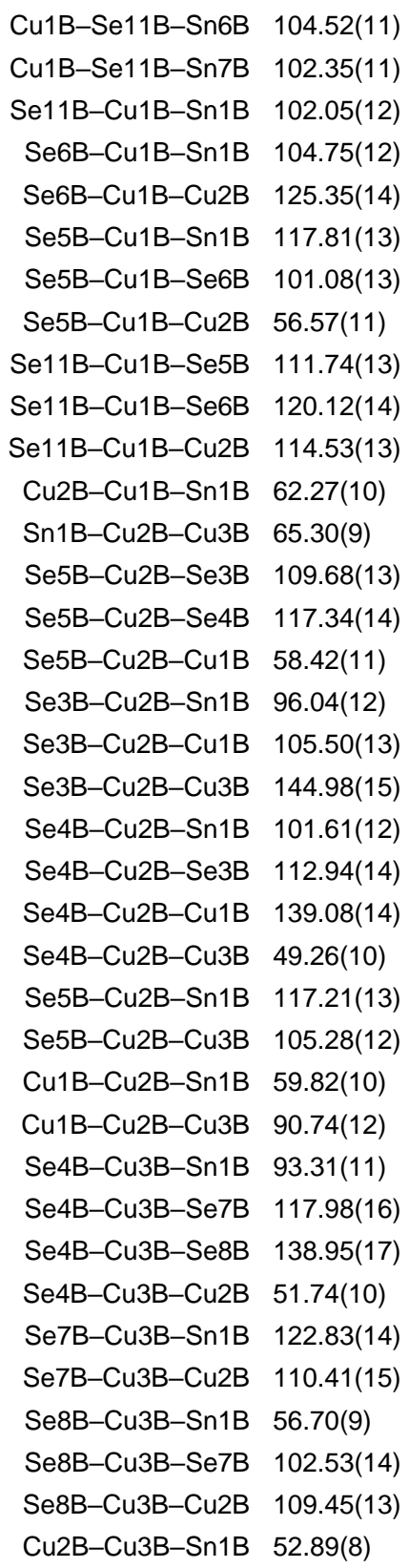




\section{Crystal Structure of 2}

Crystals suitable for X-ray diffraction analyses were investigated with a STOE IPDS-2T (2) diffractometer at $100 \mathrm{~K}$ using Mo- $K_{\alpha}$ radiation and a graphite monochromator $(\lambda=0.71073 \AA$ A). Upon numerical absorption correction (STOE X-AREA), the structure solution was performed by direct methods, followed by full-matrix-least-squares refinement against $F^{2}$, using SHELXT15, SHELXL15, and OLEX2 software. ${ }^{4-6}$

The highest peak of residual electron density on the difference Fourier map $\left(4.727 \mathrm{e}^{-} / \AA^{3}\right)$ is found $0.938 \AA$ away from

Sn4. A photograph of a single crystal of $\mathbf{2}$ is shown in Figure S7, and a cut-out of the crystal structure is shown in

Figure S8.

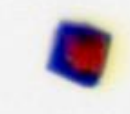

\section{$0,2 \mathrm{~mm}$}

Figure S7. Photograph of the single crystal of 2 picked for X-ray diffraction.

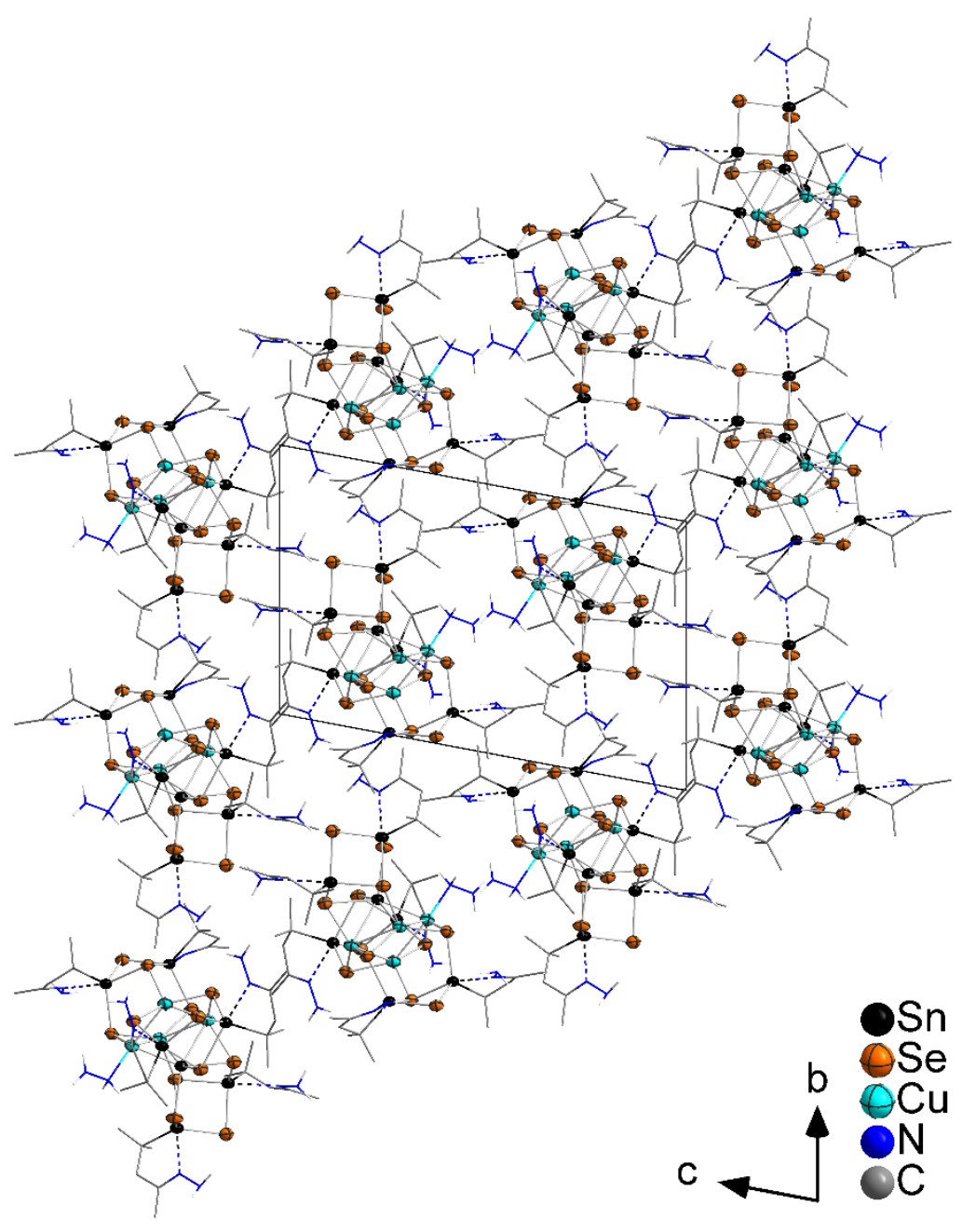

Figure S8. Excerpt of the crystal structure of $\mathbf{2}$ viewed along the a axis. 
Table S5. Crystallographic data and refinement results of 2.

\begin{tabular}{|c|c|}
\hline Compound & 2 \\
\hline Empirical Formula & $\mathrm{C}_{36} \mathrm{H}_{82} \mathrm{Cu}_{4} \mathrm{~N}_{14} \mathrm{Se}_{12} \mathrm{Sn}_{7}$ \\
\hline Formula weight $/ \mathrm{g} \cdot \mathrm{mol}^{-1}$ & 2743.66 \\
\hline Crystal color and shape & dark orange block \\
\hline Crystal size $/ \mathrm{mm}^{3}$ & $0.059 \times 0.068 \times 0.079$ \\
\hline Crystal system & triclinic \\
\hline Space group & $P \overline{1}$ \\
\hline$a / \AA$ & $13.445(3)$ \\
\hline$b / \AA$ & $14.236(3)$ \\
\hline$c / \AA$ & $20.667(4)$ \\
\hline$\alpha /{ }^{\circ}$ & $78.374(15)$ \\
\hline$\beta /{ }^{\circ}$ & $85.303(15)$ \\
\hline$y /{ }^{\circ}$ & $69.962(15)$ \\
\hline$V / \AA^{3}$ & $3639.7(12)$ \\
\hline$Z$ & 2 \\
\hline Measurement Temperature /K & 100 \\
\hline$\rho_{\text {calcd }} / \mathrm{g} \cdot \mathrm{cm}^{-3}$ & 2.503 \\
\hline$\mu_{(\mathrm{Mo} \mathrm{Ka})} / \mathrm{mm}^{-1}$ & 9.536 \\
\hline Absorption correction type & numerical \\
\hline Min./max. transmission & $0.2326 / 0.6769$ \\
\hline $2 \theta$ range / deg & $3.18-53.93$ \\
\hline No. of measured reflections & 34386 \\
\hline$R$ (int) & 0.1762 \\
\hline Independent Reflections & 15319 \\
\hline Independent Reflections $(>2 \sigma(I))$ & 7916 \\
\hline No. of parameters & 681 \\
\hline$R_{1}(\mid>2 \sigma(I)) / w R_{2}$ (all data) & $0.0969 / 0.2309$ \\
\hline$S$ (all data) & 0.883 \\
\hline Max. peak / hole / $\mathrm{e}^{-} \AA^{3}$ & $4.727 /-2.900$ \\
\hline
\end{tabular}


Table S6. Fractional Atomic Coordinates $\left(\times 10^{4}\right)$ and Equivalent Isotropic Displacement Parameters $\left(\AA^{2} \times 10^{3}\right)$ for 2 . U(eq) is defined as $1 / 3$ of of the trace of the orthogonalised $U_{I J}$ tensor.

\begin{tabular}{|c|c|c|c|c|c|c|c|c|c|}
\hline Atom & $x$ & $y$ & $z$ & $\mathrm{U}(\mathrm{eq})$ & Atom & $x$ & $y$ & $z$ & $\mathrm{U}(\mathrm{eq})$ \\
\hline Sn1 & $3484.9(10)$ & $1858.2(10)$ & $8682.9(6)$ & $40.4(3)$ & C1 & $3020(16)$ & $2032(16)$ & $9708(9)$ & $45(5)$ \\
\hline Sn2 & $2405.5(10)$ & $3183.1(10)$ & $7117.0(6)$ & $39.2(3)$ & $\mathrm{C} 2$ & $2360(20)$ & $3199(18)$ & $9722(12)$ & $64(6)$ \\
\hline Sn3 & $5066.5(10)$ & $3781.5(10)$ & $7588.7(6)$ & $39.7(3)$ & C3 & $4000(20)$ & $1712(18)$ & $10098(12)$ & $64(6)$ \\
\hline Sn4 & $5939.0(11)$ & $6126.9(10)$ & $7483.0(7)$ & $44.9(3)$ & C4 & 2289(17) & $1423(18)$ & $9902(10)$ & $57(6)$ \\
\hline Sn5 & $7109.3(10)$ & $4090.3(10)$ & $8740.7(7)$ & $42.9(3)$ & C5 & $2714(16)$ & $357(15)$ & $9769(10)$ & $44(4)$ \\
\hline Sn6 & $8579.0(10)$ & $77.7(9)$ & $7294.7(6)$ & $38.2(3)$ & C6 & $2380(20)$ & $-483(17)$ & $10181(12)$ & $57(6)$ \\
\hline Sn7 & $7571.1(10)$ & $1259.3(9)$ & $5736.1(6)$ & $38.6(3)$ & $\mathrm{C} 7$ & $1122(14)$ & $4611(14)$ & $6749(9)$ & $40(4)$ \\
\hline Se1 & $5325.7(15)$ & $861.6(14)$ & $8354.5(10)$ & $41.8(4)$ & C8 & $818(15)$ & $5349(14)$ & $7233(10)$ & $44(4)$ \\
\hline Se2 & $3159.2(14)$ & $3784.2(14)$ & $8067.9(10)$ & $40.7(4)$ & C9 & $1523(17)$ & $5108(16)$ & $6111(10)$ & $51(5)$ \\
\hline Se3 & $1998.8(15)$ & $1826.6(15)$ & $8008.2(10)$ & $42.3(4)$ & C10 & $199(18)$ & $4294(17)$ & $6663(10)$ & $52(5)$ \\
\hline $\mathrm{Se} 4$ & $4000.4(14)$ & $2449.4(14)$ & $6416.0(9)$ & $39.8(4)$ & C11 & $431(15)$ & $3363(16)$ & $6367(9)$ & $44(4)$ \\
\hline Se5 & $6966.0(14)$ & $3070.4(14)$ & $5882.4(9)$ & $39.3(4)$ & C12 & $-449(15)$ & $3172(17)$ & $6011(12)$ & $55(5)$ \\
\hline Se6 & $9464.9(15)$ & $245.1(14)$ & $6144.2(9)$ & $41.5(4)$ & C13 & $6507(17)$ & $7033(16)$ & $6627(11)$ & $48(5)$ \\
\hline Se7 & $6808.1(14)$ & $271.3(14)$ & $6762.7(9)$ & $39.0(4)$ & C14 & $6680(20)$ & $6550(20)$ & $6053(12)$ & $70(7)$ \\
\hline Se8 & $8173.4(14)$ & $1596.6(14)$ & 7837.7(9) & $39.6(4)$ & C15 & $7570(20)$ & $7050(20)$ & $6854(13)$ & $69(7)$ \\
\hline Se9 & $5678.7(15)$ & $3277.8(15)$ & $8881.9(10)$ & $42.6(4)$ & C16 & $5672(19)$ & $8127(14)$ & $6497(11)$ & $58(6)$ \\
\hline Se10 & $7392.4(15)$ & $4317.3(14)$ & $7445.8(10)$ & $41.4(4)$ & C17 & $4929(19)$ & $8461(15)$ & $7038(12)$ & $57(6)$ \\
\hline Se11 & $6378.5(17)$ & $6005.6(16)$ & $8693.8(10)$ & $49.6(5)$ & C18 & $4239(17)$ & $9584(13)$ & $6951(13)$ & $58(6)$ \\
\hline Se12 & $4246.1(16)$ & $5777.4(15)$ & $7423.7(11)$ & $49.0(5)$ & C19 & $8688(16)$ & $3547(17)$ & $9163(10)$ & $50(5)$ \\
\hline Cu1 & $6420.1(18)$ & $1888.3(18)$ & $8232.4(12)$ & $42.8(5)$ & $\mathrm{C} 20$ & $9422(16)$ & $3852(17)$ & $8668(11)$ & $53(5)$ \\
\hline Cu2 & $5386.0(18)$ & $1579.9(19)$ & 7199.1(12) & $46.1(6)$ & $\mathrm{C} 21$ & $9059(17)$ & $2367(15)$ & $9352(11)$ & $50(5)$ \\
\hline Cu3 & $5228.3(18)$ & $3379.2(18)$ & $6371.7(11)$ & $40.8(5)$ & $\mathrm{C} 22$ & $8623(18)$ & $3959(19)$ & $9780(11)$ & $58(6)$ \\
\hline Cu4 & 6998.3(18) & $2926.9(17)$ & $7053.0(11)$ & $41.5(5)$ & $\mathrm{C} 23$ & $7740(18)$ & $3902(16)$ & $10244(11)$ & $51(5)$ \\
\hline N1 & $4430(15)$ & $4831(13)$ & $5798(9)$ & $55(4)$ & $\mathrm{C} 24$ & $7780(20)$ & $4022(17)$ & $10958(11)$ & $66(7)$ \\
\hline N2 & 3992(15) & $4679(15)$ & $5241(9)$ & $61(5)$ & $\mathrm{C} 25$ & $8945(16)$ & $-1359(15)$ & $8022(10)$ & $51(5)$ \\
\hline N3 & $3368(13)$ & $228(12)$ & $9275(8)$ & $47(4)$ & $\mathrm{C} 26$ & 7898(15) & $-1469(14)$ & $8304(10)$ & $47(5)$ \\
\hline N4 & $3787(16)$ & $-657(14)$ & $9066(9)$ & $62(5)$ & $\mathrm{C} 27$ & $9560(16)$ & $-2265(13)$ & $7671(10)$ & $48(5)$ \\
\hline N5 & $1378(12)$ & $2738(12)$ & $6425(8)$ & $43(4)$ & $\mathrm{C} 28$ & $9573(15)$ & $-1230(14)$ & $8523(10)$ & $43(4)$ \\
\hline N6 & $1642(13)$ & $1798(11)$ & $6219(8)$ & $44(4)$ & $\mathrm{C} 29$ & $10491(16)$ & $-851(17)$ & $8267(11)$ & $51(5)$ \\
\hline N7 & $4881(17)$ & $7801(14)$ & $7549(10)$ & $66(6)$ & $\mathrm{C} 30$ & $11310(20)$ & $-950(20)$ & $8745(13)$ & $73(7)$ \\
\hline N8 & $4170(20)$ & $8123(19)$ & $8048(13)$ & $110(11)$ & C31 & $6828(16)$ & $905(15)$ & $4944(9)$ & $44(4)$ \\
\hline N9 & $6909(15)$ & $3831(13)$ & $10021(9)$ & $52(4)$ & C32 & $5718(16)$ & $997(17)$ & $5144(10)$ & $53(5)$ \\
\hline N10 & $6076(17)$ & $3737(19)$ & $10440(9)$ & $71(6)$ & C33 & 7491(18) & $-196(17)$ & $4871(11)$ & $54(5)$ \\
\hline N11 & $10427(13)$ & $-383(12)$ & $7668(7)$ & $41(4)$ & C34 & $6889(14)$ & $1663(17)$ & $4319(10)$ & $47(5)$ \\
\hline N12 & $11206(14)$ & $-9(12)$ & $7401(9)$ & $49(4)$ & C35 & $7937(14)$ & $1867(14)$ & $4201(10)$ & $44(4)$ \\
\hline N13 & $8476(12)$ & $1717(11)$ & $4704(7)$ & $39(4)$ & $\mathrm{C} 36$ & $8242(16)$ & $2235(15)$ & $3514(10)$ & $48(5)$ \\
\hline N14 & $9433(13)$ & $1920(12)$ & $4652(9)$ & $46(4)$ & & & & & \\
\hline
\end{tabular}


Table S7. Selected bond lengths $[\AA]$ in 2.

\begin{tabular}{|c|c|c|c|c|c|}
\hline Sn1-Se1 & $2.511(2)$ & $\mathrm{Sn} 4-\mathrm{Se} 12$ & $2.508(3)$ & Se1-Cu2 & $2.407(3)$ \\
\hline Sn1-Se2 & $2.681(2)$ & Sn4-N7 & $2.345(17)$ & Se4-Cu2 & $2.393(3)$ \\
\hline Sn1-Se3 & $2.546(2)$ & Sn4-C13 & $2.22(2)$ & Se4-Cu3 & $2.431(3)$ \\
\hline Sn1-N3 & $2.446(16)$ & Sn5-Se9 & $2.534(2)$ & Se5-Cu3 & $2.401(3)$ \\
\hline Sn1-C1 & $2.198(18)$ & Sn5-Se10 & $2.642(2)$ & Se5-Cu4 & $2.389(3)$ \\
\hline $\mathrm{Sn} 2-\mathrm{Se} 2$ & $2.687(2)$ & Sn5-Se11 & $2.546(2)$ & Se7-Cu2 & $2.428(3)$ \\
\hline $\mathrm{Sn} 2-\mathrm{Se} 3$ & $2.558(2)$ & Sn5-C19 & $2.18(2)$ & Se8-Cu1 & $2.355(3)$ \\
\hline Sn2-Se4 & $2.521(2)$ & Sn6-Se6 & $2.575(2)$ & Se8-Cu4 & $2.417(3)$ \\
\hline Sn2-N5 & $2.376(17)$ & Sn6-Se7 & $2.610(2)$ & Se9-Cu1 & $2.492(3)$ \\
\hline Sn2-C7 & $2.208(18)$ & Sn6-Se8 & $2.510(2)$ & Se10-Cu4 & $2.509(3)$ \\
\hline Sn3-Se2 & $2.671(2)$ & Sn6-N11 & $2.489(16)$ & Cu1-Cu2 & $2.823(4)$ \\
\hline Sn3-Se9 & $2.742(2)$ & Sn6-C25 & $2.21(2)$ & Cu1-Cu4 & $2.785(3)$ \\
\hline Sn3-Se12 & $2.632(2)$ & Sn7-Se5 & $2.497(2)$ & Cu2-Cu3 & $2.730(3)$ \\
\hline Sn3-Cu1 & $2.817(3)$ & Sn7-Se6 & $2.573(2)$ & Cu3-Cu4 & $2.682(3)$ \\
\hline Sn3-Cu3 & $2.666(3)$ & Sn7-Se7 & $2.638(2)$ & Cu3-N1 & $2.128(19)$ \\
\hline Sn3-Cu4 & $2.713(3)$ & Sn7-N13 & $2.453(14)$ & $\mathrm{N} 1-\mathrm{N} 2$ & $1.42(3)$ \\
\hline Sn4-Se10 & $2.658(2)$ & Sn7-C31 & $2.208(19)$ & & \\
\hline Sn4-Se11 & $2.577(3)$ & Se1-Cu1 & $2.373(3)$ & & \\
\hline
\end{tabular}

Table S8. Selected bond angles $\left[^{\circ}\right]$ in 2.

\begin{tabular}{|c|c|c|c|c|c|}
\hline Se1-Sn1-Se2 & $105.67(8)$ & Se12-Sn3-Se2 & $85.49(7)$ & Se9-Sn5-Se10 & $100.13(8)$ \\
\hline Se1-Sn1-Se3 & $115.66(8)$ & Se12-Sn3-Se9 & $101.74(8)$ & Se9-Sn5-Se11 & $113.04(8)$ \\
\hline Se3-Sn1-Se2 & $88.72(8)$ & Se12-Sn3-Cu1 & $152.08(9)$ & Se11-Sn5-Se10 & $92.14(8)$ \\
\hline N3-Sn1-Se1 & $86.2(4)$ & Se12-Sn3-Cu3 & $104.53(9)$ & Se6-Sn6-Se7 & $90.56(7)$ \\
\hline N3-Sn1-Se2 & $167.6(4)$ & Se12-Sn3-Cu4 & $119.15(8)$ & Se8-Sn6-Se6 & $114.99(8)$ \\
\hline N3-Sn1-Se3 & $83.0(4)$ & Cu3-Sn3-Se2 & $107.30(8)$ & Se8-Sn6-Se7 & $105.42(7)$ \\
\hline $\mathrm{C} 1-\mathrm{Sn} 1-\mathrm{Se} 1$ & $122.9(6)$ & Cu3-Sn3-Se9 & $151.35(8)$ & N11-Sn6-Se6 & $82.4(3)$ \\
\hline C1-Sn1-Se2 & $102.4(6)$ & Cu3-Sn3-Cu1 & $98.45(8)$ & N11-Sn6-Se7 & $168.4(3)$ \\
\hline $\mathrm{C} 1-\mathrm{Sn} 1-\mathrm{Se} 3$ & $113.6(6)$ & Cu3-Sn3-Cu4 & $59.82(8)$ & N11-Sn6-Se8 & $86.0(4)$ \\
\hline C1-Sn1-N3 & $72.9(7)$ & Cu4-Sn3-Se9 & $97.19(8)$ & C25-Sn6-Se6 & $123.8(4)$ \\
\hline Se3-Sn2-Se2 & $88.33(8)$ & Cu4-Sn3-Cu1 & $60.45(7)$ & C25-Sn6-Se7 & $104.8(6)$ \\
\hline $\mathrm{Se} 4-\mathrm{Sn} 2-\mathrm{Se} 2$ & $104.89(8)$ & Se11-Sn4-Se10 & $91.08(8)$ & C25-Sn6-Se8 & 112.1(5) \\
\hline $\mathrm{Se} 4-\mathrm{Sn} 2-\mathrm{Se} 3$ & $112.64(8)$ & Se12-Sn4-Se10 & $102.25(8)$ & C25-Sn6-N11 & $72.1(7)$ \\
\hline N5-Sn2-Se2 & $166.9(4)$ & Se12-Sn4-Se11 & $110.39(9)$ & Se5-Sn7-Se6 & $113.19(8)$ \\
\hline N5-Sn2-Se3 & $84.8(4)$ & N7-Sn4-Se10 & $170.9(6)$ & Se5-Sn7-Se7 & $105.99(7)$ \\
\hline N5-Sn2-Se4 & $88.1(4)$ & N7-Sn4-Se11 & $84.6(5)$ & Se6-Sn7-Se7 & $89.97(7)$ \\
\hline C7-Sn2-Se2 & $99.4(5)$ & N7-Sn4-Se12 & $86.7(6)$ & N13-Sn7-Se5 & $89.6(3)$ \\
\hline C7-Sn2-Se3 & $119.7(5)$ & C13-Sn4-Se10 & $98.7(5)$ & N13-Sn7-Se6 & 83.7(4) \\
\hline C7-Sn2-Se4 & $122.3(5)$ & C13-Sn4-Se11 & $124.1(6)$ & N13-Sn7-Se7 & $164.4(3)$ \\
\hline C7-Sn2-N5 & $74.6(6)$ & C13-Sn4-Se12 & $120.6(6)$ & C31-Sn7-Se5 & $117.2(6)$ \\
\hline Se2-Sn3-Se9 & $85.86(7)$ & C13-Sn4-N7 & 77.3(7) & C31-Sn7-Se6 & 123.8(6) \\
\hline Se2-Sn3-Cu1 & $102.73(8)$ & C19-Sn5-Se9 & $130.6(6)$ & C31-Sn7-Se7 & $98.6(5)$ \\
\hline Se2-Sn3-Cu4 & $153.59(8)$ & C19-Sn5-Se10 & $106.2(6)$ & C31-Sn7-N13 & $73.6(6)$ \\
\hline Se9-Sn3-Cu1 & $53.24(7)$ & C19-Sn5-Se11 & $107.0(6)$ & Cu1-Se1-Sn1 & $109.64(9)$ \\
\hline
\end{tabular}




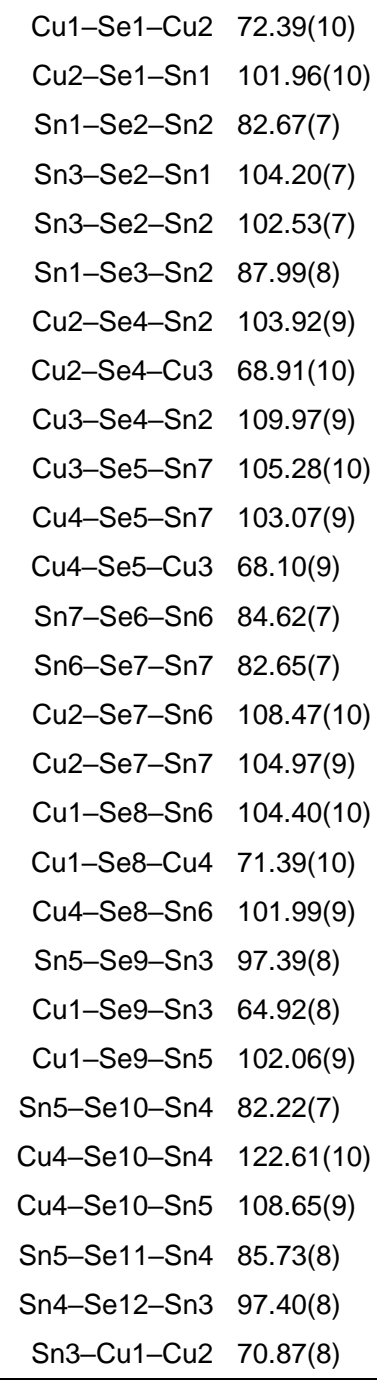

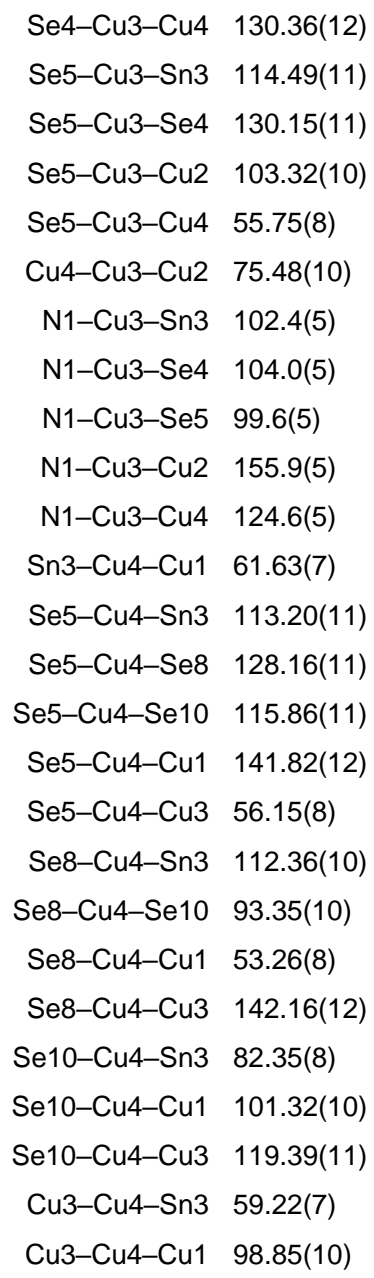

\section{References for the Supporting Information}

(1) Rinn, N.; Eußner, J. P.; Kaschuba, W.; Xie, X.; Dehnen, S. Formation and Reactivity of Organo-Functionalized Tin Selenide Clusters. Chem. Eur. J. 2016, 22, 3094-3104.

(2) Detty, M. R.; Seidler, M. D. Bis(trialkylsilyl) chalcogenides. 1. Preparation and reduction of group VIA oxides. J. Org. Chem. 1982, 47, 1354-1356.

(3) Hanna, J. V.; Boyd, S. E.; Healy, P. C.; Bowmaker, G. A.; Skelton, B. W.; White, A. H. Structural and solid state ${ }^{31} \mathrm{P}$ NMR studies of the four-coordinate copper(I) complexes $\left[\mathrm{Cu}\left(\mathrm{PPh}_{3}\right)_{3} \mathrm{X}\right]$ and $\left[\mathrm{Cu}\left(\mathrm{PPh}_{3}\right)_{3}\left(\mathrm{CH}_{3} \mathrm{CN}\right)\right] \mathrm{X}$. Dalton Trans. 2005, 2547-2556.

(4) Sheldrick, G. M. Crystal structure refinement with SHELXL. Acta Cryst., Sect. C: Struct. Chem. 2015, 71, 3-8.

(5) Sheldrick, G. M. SHELXT - integrated space-group and crystal-structure determination. Acta Cryst., Sect. A: Found. Adv. 2015, 71, 3-8.

(6) Dolomanov, O. V.; Bourhis, L. J.; Gildea, R. J.; Howard, J. A. K.; Puschmann, H. OLEX2: A complete structure solution, refinement and analysis program. J. Appl. Crystallogr. 2009, 42, 339-341.

(7) Spek, A. L. PLATON SQUEEZE: a tool for the calculation of the disordered solvent contribution to the calculated structure factors. Acta Crystallogr., Sect. C: Struct. Chem. 2015, 71, 9-18. 